\title{
ON THE LENGTH OF GAPS IN THE ESSENTIAL SPECTRUM OF A GENERALISED DIRAC OPERATOR
}

\author{
W. D. EVANS 1
}

\begin{abstract}
Abstracr. The object of the paper is to give an upper bound for the length of the gaps that can occur in the essential spectrum of any selfadjoint operator which is generated by a generalised Dirac system of differential expressions in the Hilbert space $L^{2}(a, b)$. An estimate is also obtained for the limit point of the spectrum which has least absolute value.
\end{abstract}

1. Let $\tau$ denote the matrix differential expression of order $2 n$ given by

$$
\tau \varphi(t)=B \varphi^{\prime}(t)+\Omega(t) \varphi(t), \quad-\infty \leqq a<t<b \leqq \infty,
$$

where $B$ is the real $2 n \times 2 n$ matrix

$$
B=\left(\begin{array}{ll} 
& I \\
-I &
\end{array}\right), \quad I=\left(\begin{array}{lll} 
& & 1 \\
& . & \\
. & &
\end{array}\right)
$$

$\Omega(t)$ is a real symmetric $2 n \times 2 n$ matrix for each $t \in(a, b)$ and $q$ is a $C^{2 n_{-}}$ valued function on $(a, b)$ having a derivative $q^{\prime}$. Our aim in this paper is to study the selfadjoint operators which are generated by $\tau$ in the Hilbert space $L_{2 \prime \prime}^{2}(a, b)$ of $C^{2 n}$-valued Lebesgue measurable functions $q$ which satisfy

$$
\|p\|^{2}=\int_{a}^{b}|\eta(t)|^{2} d t<\infty
$$

Received by the editors November 22, 1971.

AMS 1970 subject classifications. Primary 34A30, 34B25, Secondary 47A10, 47B25.

Key words and phrases. Selfadjoint operator, Hilbert space, deficiency indices, essential spectrum.

${ }^{1}$ The author wishes to thank the Canadian National Research Council for the opportunity to visit the University of Toronto during the session 1971-1972 and also the United Kingdom Science Research Council for a travel grant.

1. American Mathematical Society 1972 
$|\cdot|$ denoting the Euclidean norm on $C^{2 n}$. The inner product on $L_{2 n}^{2}(a, b)$ is written

$$
(\varphi, \psi)=\int_{a}^{b} \varphi(t) \cdot \psi(t) d t
$$

where $\varphi(t) \cdot y^{\prime}(t)$ is the inner product $\sum_{i=1}^{2 n} \gamma_{i}(t) \bar{\psi}_{i}(t)$ on $C^{2 n}$.

Let $T_{0}$ be the operator defined by $T_{0} q=\tau \varphi$ on the space of $C^{2 n}$-valued functions $q$ : which have a continuous first derivative in $(a, b)$ and whose support is a compact subset of the open interval $(a, b)$. For a suitable $\Omega$ this operator $T_{0}$ is a symmetric operator in $L_{2 n}^{2}(a, b)$ as $\tau$ is formally selfadjoint, and the closure of $T_{0}$ is the minimal operator generated by $\tau$.

The expression $\tau$ can be singular at one or both of the end points of $(a, b)$. However our discussion does require the interval to be infinite and so it will always be assumed that at least one of the end points is infinite. In every case the equation $(\tau-\lambda) \varphi=0$ has only a finite number of $L_{2 n}^{2}(a, b)$ solutions for im $\lambda \neq 0$ which means that $T_{0}$ has finite (and of course equal) deficiency indices. This in turn implies that every selfadjoint extension $T$ of $T_{0}$ has the same essential spectrum $\sigma_{F}$. Our concern in this paper is with the nature of this essential spectrum, in particular with determining bounds for the length of any gap that can occur in $\sigma_{E}$. We also obtain a bound for the limit point of $\sigma_{E}$ which is nearest the origin.

In [4] Weidmann, inter alia, obtains results on the essential spectrum of any selfadjoint extension $T$ of $T_{0}$ in the case when $n=1, \tau$ is regular at $a$ and $b=\infty$. As a corollary to our main theorem we obtain a result which is similar to Theorem 6.10 in [4]. Unlike that of Weidmann our result holds for any infinite interval $(a, b)$ and the conditions on $\Omega$ are only imposed in a sequence of intervals in $(a, b)$ whose lengths tend to infinity. However, although it is easy to find matrices $\Omega$ which satisfy our criterion and not Weidmann's our result is not stronger than his. A more detailed discussion of these results will be postponed until $\S 2$. The general system in this paper was studied by Gasymov in [3] in his work on the inverse scattering problem but with $a=0, b=\infty$. In order to give a satisfactory solution to the inverse scattering problem Gasymov assumes that the matrix-valued function $\Omega$ is $B$ selfadjoint, i.e. for any $t \in(a, b), B \Omega(t)$ is a symmetric matrix. This is equivalent to taking $\Omega$ in the form

$$
\Omega=\left(\begin{array}{cc}
P & Q I \\
I Q & -I P I
\end{array}\right)
$$

where $P(t), Q(t)$ are real symmetric $n \times n$ matrices. He does however show that the problem with a general $\Omega$ is unitarily equivalent to one with a $B$ selfadjoint $\Omega$ if $|\Omega|$, the norm of $\Omega$ in $C^{2 n}$, is assumed to be integrable over $[0, \infty)$. This integrability condition on $\Omega$ is too strong for our needs, 
although we shall make use of the notion of $B$ selfadjointness in our discussion.

2. Before we state and prove our main results it is convenient at this stage to collect some simple facts about $B$ selfajdoint matrices.

Since $B^{*}=-B$, a matrix $\Omega_{0}$ is $B$ selfadjoint if and only if $\Omega_{0} B=-B \Omega_{0}$. Hence, since $\left(\Omega_{0}+\lambda\right) B v=-B\left(\Omega_{0}-\lambda\right) v$ and $B$ is nonsingular $\left(B^{2}=-E_{2 n}\right)$, it follows that if $\lambda$ is an eigenvalue of $\Omega_{0}$ with eigenvector $v$, then $-\lambda$ is also an eigenvalue of $\Omega_{0}$ with eigenvector $B v$. The eigenvalues of $\Omega_{0}$ can thus be denoted $\pm \mu_{i}, i=1,2, \cdots, n$ (repeated according to multiplicity). If $e_{i}$ is a normalised eigenvector of $\Omega_{0}$ corresponding to $\mu_{i}, B e_{i}$ is a normalised eigenvector for $-\mu_{i}$. Suppose that the set $\left\{e_{1}, \cdots, e_{n}, B e_{1}, \cdots, B e_{n}\right\}$ is orthonormal. The unitary matrix $U=\left(e_{1}, \cdots, e_{n}, B e_{n}, \cdots, B e_{1}\right)$ diagonalises $\Omega_{0}$,

$$
U^{*} \Omega_{0} U=d g\left(\mu_{1}, \cdots, \mu_{n},-\mu_{n}, \cdots,-\mu_{1}\right)
$$

and further $B U=-U B$ so that

$$
U^{*} B U=-B .
$$

Lastly, if $\Omega$ is any real symmetric matrix then $\Omega+B \Omega B$ is $B$ selfadjoint.

Henceforth we shall denote by $T$ any selfajdoint extension of $T_{0}$ and we denote by $\left(\Lambda, \Lambda^{\prime}\right)$ a gap in the essential spectrum $\sigma_{E}$ of $T$. We shall also write $\xi_{i}$ for the vector $\left\{\delta_{j i}\right\}$ in $C^{2 n}$, where $\delta_{j i}$ is the Kronecker delta. We assume throughout that $|\Omega|$ is square integrable on a sequence of intervals $A_{m}$.

THEOREM 1. Let $A_{m}=\left[c_{m}-a_{m}, c_{m}+a_{m}\right]$ be intervals in $(a, b)$ with $a_{m} \rightarrow \infty$ as $m \rightarrow \infty$. Let $\Omega_{0}$ be a real $B$ selfadjoint constant matrix with eigenvalues $\pm \mu_{j}(j=1,2, \cdots, n), \mu_{1} \leqq \mu_{2} \leqq \cdots \leqq \mu_{n}$, and orthonormal eigenvectors $e_{j}, B e_{j}, j=1,2, \cdots, n$.

If $\frac{1}{2}\left(\Lambda^{\prime}+\Lambda\right) \in \mathrm{C}\left(-\mu_{j}, \mu_{j}\right)$ then

$$
\Lambda^{\prime}-\Lambda \leqq 2 \liminf _{m \rightarrow \infty} \frac{1}{\left(2 a_{m}\right)^{1 / 2}}\left\|\left\{\left|\left(\Omega-\Omega_{0}\right) e_{j}\right|+\left|\left(\Omega-\Omega_{0}\right) B e_{j}\right|\right\}\right\|_{A_{m}}
$$

so that $\sigma_{E} \supset \mathrm{C}\left(-\mu_{j}, \mu_{j}\right)$ if

$$
\liminf _{m \rightarrow \infty} \frac{1}{\left(2 a_{m}\right)^{1 / 2}} \|\left\{\left|\left(\Omega-\Omega_{0}\right) e_{j}\right|+\left|\left(\Omega-\Omega_{0}\right) B e_{j}\right|\right\} A_{m}=0 .
$$

If $\frac{1}{2}\left(\Lambda^{\prime}+\Lambda\right) \in\left(-\mu_{j}, \mu_{j}\right)$ then

$$
\begin{aligned}
& \Lambda^{\prime} \leqq \mu_{j}+\liminf _{m \rightarrow \infty} \frac{1}{\left(2 a_{m}\right)^{1 / 2}}\left\|\left(\Omega-\Omega_{0}\right) e_{j}\right\|_{1_{m}{ }^{\prime}} \\
& \Lambda \geqq-\mu_{j}-\liminf _{m \rightarrow \infty} \frac{1}{\left(2 a_{m}\right)^{1 / 2}}\left\|\left(\Omega-\Omega_{0}\right) B e_{j}\right\|_{\Lambda_{m}} .
\end{aligned}
$$


Note that if $\Omega_{n}$ is diagonal and $\mu_{j}$ is in the $s$ th place along the diagonal then $e_{j}=\xi_{s}, B e_{j}= \pm \xi_{2 n+1-s}$. Also, the latter results give a bound for the closest point of $\sigma_{E}$ to the origin. For if $\Lambda_{0}$ is such a point the gap $\left(-|\Lambda|_{0},\left|\Lambda_{0}\right|\right)$ in $\sigma_{K}$ has midpoint 0 and so

$$
\begin{aligned}
\left|\Lambda_{0}\right| \leqq \min _{1 \subseteq j \leqq n}\left\{\mu_{j}+\min \left(\liminf _{m \rightarrow \infty}\right.\right. & \frac{1}{\left(2 a_{m}\right)^{1 / 2}}\left\|\left(\Omega-\Omega_{0}\right) e_{j}\right\|_{A_{m}} \\
& \left.\left.\liminf _{m \rightarrow \infty} \frac{1}{\left(2 a_{m}\right)^{1 / 2}}\left\|\left(\Omega-\Omega_{0}\right) B e_{j}\right\|_{A_{m}}\right)\right\} .
\end{aligned}
$$

Proof. We have remarked above that the constant unitary matrix $U=\left(e_{1}, \cdots, e_{n}, B e_{n}, \cdots, B e_{1}\right)$ satisfies

$$
U^{*} \Omega_{0} U=d g\left(\mu_{1}, \cdots, \mu_{n},-\mu_{n}, \cdots,-\mu_{1}\right)=D
$$

say, and $U^{*} B U=-B$. Hence $T$ is unitarily equivalent to the operator $\tilde{T}$ which is generated by $\tilde{\tau}$, where

$$
\tilde{\tau}_{\varphi}=U^{*} \tau U^{\prime} \varphi^{\prime}=-B \varphi^{\prime}+D \varphi+U^{*}\left(\Omega-\Omega_{0}\right) U \varphi .
$$

It is therefore sufficient to prove the result for $\tilde{T}$.

The proof depends on the construction of a sequence of $C^{2 n}$-valued functions $\phi^{(m)}$ which lie in the domain of $T_{0}$ and converge weakly to zero. The idea behind such a sequence comes from the work of Eastham on similar problems concerning single differential expressions (see [1], [2]).

Let $u_{m}$ be a real-valued function having a continuous first derivative and satisfying

$$
\begin{aligned}
& u_{m}(t)=1 \text { for }|t| \leqq a_{m}-1 \text {, } \\
& =0 \text { for }|t| \geqq a_{m},
\end{aligned}
$$

and $0 \leqq u_{n}(t) \leqq 1$ for all $t$. Let $\lambda=\frac{1}{2}\left(\Lambda^{\prime}+\Lambda\right)$ and take $|\lambda|>\mu_{j}$. We define

$$
\begin{aligned}
\varphi^{(m)}\left(t, \lambda_{1}\right) & =k_{m} \exp \left(i f_{m}(t, \lambda)\right) u_{m}\left(t-c_{m}\right) v \text { for }\left|t-c_{m}\right| \leqq a_{m}, \\
& =0 \text { otherwise, }
\end{aligned}
$$

where $k_{m}$ is a positive real constant making $\left\|\varphi^{(m)}\right\|=1, f_{m}$ is a real-valued function and $v^{\prime}$ a vector in $C^{2 n}$ with $|v|=1 ; f_{m}$ and $v$ will be chosen later. We have, as $m \rightarrow x$.

$$
k_{m} \sim\left(2 a_{m}\right)^{1 / 2}, \quad\left|\varphi^{(m)}(t, \lambda)\right| \leqq k_{m} .
$$

From these equations it follows that $q^{(m)} \rightarrow 0$ as $m \rightarrow \infty$.

Now,

$$
\begin{aligned}
\left(\tilde{\tau}-\lambda . \varphi^{(m)}=\right. & \left(-i f_{m}^{\prime} B+D-\lambda\right) \varphi^{(m)} \\
& -k_{m} \exp \left(i f_{m}\right) u_{m}^{\prime}\left(\cdot-c_{m}\right) B v+U^{*}\left(\Omega-\Omega_{0}\right) U \varphi^{(m)} .
\end{aligned}
$$


We choose $f_{m}$ and $v$ to satisfy

It is readily shown that

$$
\left(-i f_{m}^{\prime} B+D-\lambda\right) v=0
$$

$$
\operatorname{det}\left(-i f_{m}^{\prime} B+D-\lambda\right)=\prod_{j=1}^{n}\left\{\left(\lambda^{2}-\mu_{j}^{2}\right)-\left(f_{m}^{\prime}\right)^{2}\right\}
$$

and so we may choose $\left(f_{m}^{\prime}\right)^{2}=\lambda^{2}-\mu_{j}^{2}$ and $f_{m}(t, \lambda)=\left(\lambda^{2}-\mu_{j}^{2}\right)^{1 / 2}\left(t-c_{m}+a_{m}\right)$ for $t \in A_{m}$ and $f_{m}(t, \lambda)=0$ otherwise. As $|\lambda|>\mu_{j}, f_{m}$ is real. For $v$ we may chouse $v=\left\{v_{i}\right\}$ where $v_{i}=0$ for $i \neq j, 2 n+1-j$ and

$$
\begin{aligned}
\left(\mu_{j}-\lambda\right) v_{j}-i f_{m}^{\prime} v_{2 n+1-j} & =0 \\
-\left(\mu_{j}+\lambda\right) v_{2 n+1-j}+i f_{m}^{\prime} v_{j} & =0 .
\end{aligned}
$$

Let $v_{j}=\left(\left(\lambda+\mu_{j}\right) / 2|\lambda|\right)^{1 / 2}, \quad v_{2 n+1-j}=\left(\left(\mu_{j}-\lambda\right) / 2|\lambda|\right)^{1 / 2}$, so that $|v|=1$ as required. Note that

so that

$$
U v=v_{j} U \xi_{j}+v_{2 n+1-j} U \xi_{2 n+1-j}=v_{j} e_{j}+v_{2 n+1-j} B e_{j}
$$

$$
\left|U^{*}\left(\Omega-\Omega_{0}\right) U v\right|=\left|\left(\Omega-\Omega_{0}\right) U v\right| \leqq\left|\left(\Omega-\Omega_{0}\right) e_{j}\right|+\left|\left(\Omega-\Omega_{0}\right) B e_{j}\right| .
$$

Substituting in (2.8) and using (2.7), we get, since $q^{(m)}$ is continuously differentiable with support in $A_{m}$,

$$
\begin{aligned}
\left\|(\tilde{T}-\lambda) \varphi^{(m)}\right\|= & \left\|(\tilde{\tau}-\lambda) \varphi^{(m)}\right\| \leqq k_{m}\left\|u_{m}^{\prime}\left(\cdot-c_{m}\right)\right\|_{A_{m}} \\
& +k_{m}\left\|\left\{\left|\left(\Omega-\Omega_{0}\right) e_{j}\right|+\left|\left(\Omega-\Omega_{0}\right) B e_{j}\right|\right\}\right\|_{A_{m}} \\
= & O\left(k_{m}\right)+k_{m}\left\|\left\{\left|\left(\Omega-\Omega_{0}\right) e_{j}\right|+\left|\left(\Omega-\Omega_{0}\right) B e_{j}\right|\right\}\right\|_{A_{m}} .
\end{aligned}
$$

As $\sigma_{E}$ has a gap $\left(\Lambda, \Lambda^{\prime}\right)$ then for any $\delta, 0<\delta<\frac{1}{2}\left(\Lambda^{\prime}-\Lambda\right),\left(\Lambda+\delta, \Lambda^{\prime}-\delta\right)$ contains only a finite number of eigenvalues of finite multiplicity of $T$ and hence also of $\tilde{T}$. Let $\lambda_{1}, \cdots, \lambda_{N}$ be these eigenvalues of $\tilde{T}$, repeated according to multiplicity, and let $\psi_{1}, \cdots, \psi_{N}$ be an orthonormal set of eigenvectors of $\tilde{T}$. If $\left\{E_{t}\right\}$ denotes the spectral family of $\tilde{T}$,

$$
\begin{aligned}
\left\|(\tilde{T}-\lambda) \varphi^{(m)}\right\|^{2}= & \int_{-\infty}^{\infty}(\lambda-t)^{2} d\left(E_{t} q^{(m)}, q^{(m)}\right) \\
= & \int_{-\infty}^{\Lambda+\delta}+\int_{\Lambda^{\prime}-\delta}^{\infty}+\int_{\Lambda+\delta}^{\Lambda^{\prime}-\delta}(\lambda-t)^{2} d\left(E_{t} q^{(m)} \cdot q^{(m)}\right) \\
\geqq & \left\{\frac{1}{2}\left(\Lambda^{\prime}-\Lambda\right)-\delta\right\}^{2}\left\{1-\sum_{i=1}^{N}\left|\left(q^{(m)}, \psi_{i}\right)\right|^{2}\right\} \\
& +\sum_{i=1}^{N}\left(\lambda-\lambda_{i}\right)^{2}\left|\left(q^{(m)}, \psi_{i}\right)\right|^{2}
\end{aligned}
$$


Since $\left(\varphi^{(m)}, \psi_{i}\right) \rightarrow 0$ as $m \rightarrow \infty, i=1,2, \cdots, N$,

$$
\Lambda^{\prime}-\Lambda \leqq 2 \delta+2 \liminf _{m \rightarrow \infty}\left\|(\tilde{T}-\lambda) \varphi^{(m)}\right\|
$$

and the result follows from (2.9) and (2.7) since $\delta$ is arbitrary.

If $\lambda \in\left(-\mu_{j}, \mu_{j}\right)$ we replace $\lambda$ by $\mu_{j}$ in $\varphi^{(m)}$ above so that $f_{m}=0, v=\xi_{j}$ and $\left(\Omega-\Omega_{0}\right) U v=\left(\Omega-\Omega_{0}\right) e_{j}$. Thus as above

$$
\begin{aligned}
\Lambda^{\prime}-\Lambda & \leqq 2 \liminf _{m \rightarrow \infty}\left\|(\tilde{T}-\lambda) \varphi^{(m)}\right\| \\
& \leqq 2 \liminf _{m \rightarrow \infty}\left\|\left(\tilde{T}-\mu_{j}\right) \varphi^{(m)}\right\|+2\left(\mu_{j}-\lambda\right)
\end{aligned}
$$

or

$$
\Lambda^{\prime} \leqq \mu_{j}+\liminf _{m \rightarrow \infty} \frac{1}{\left(2 a_{m}\right)^{1 / 2}}\left\|\left(\Omega-\Omega_{0}\right) e_{j}\right\|_{A_{m}} .
$$

Similarly, we obtain the lower bound for $\Lambda$ by replacing $\lambda$ by $-\mu_{j}$ in $\varphi^{(m)}$.

THEOREM 2. Let $A_{m}$ be as in Theorem 1 and suppose $n=1$. Let $\Omega_{0}$ be a constant, real symmetric matrix with eigenvalues $\lambda_{1}, \lambda_{2}, \lambda_{1} \leqq \lambda_{2}$, and eigenvectors $e_{1}, e_{2}$.

Then, if $\frac{1}{2}\left(\Lambda^{\prime}+\Lambda\right) \in \mathrm{C}\left(\lambda_{1}, \lambda_{2}\right)$,

$$
\Lambda^{\prime}-\Lambda \leqq 2 \liminf _{m \rightarrow \infty} \frac{1}{\left(2 a_{m}\right)^{1 / 2}}\left\|\left|\Omega-\Omega_{0}\right|\right\|_{A_{m}},
$$

so that $\sigma_{E} \supset \mathrm{C}\left(\lambda_{1}, \lambda_{2}\right)$ if

$$
\liminf _{m \rightarrow \infty} \frac{1}{\left(2 a_{m}\right)^{1 / 2}}\left\|\left|\Omega-\Omega_{0}\right|\right\|_{A_{m}}=0
$$

$$
\text { If } \frac{1}{2}\left(\Lambda^{\prime}+\Lambda\right) \in\left(\lambda_{1}, \lambda_{2}\right)
$$

$$
\begin{aligned}
& \Lambda^{\prime} \leqq \lambda_{2}+\liminf _{m \rightarrow \infty} \frac{1}{\left(2 a_{m}\right)^{1 / 2}}\left\|\left(\Omega-\Omega_{0}\right) e_{2}\right\|_{A_{m}}, \\
& \Lambda \geqq \lambda_{1}-\liminf _{m \rightarrow \infty} \frac{1}{\left(2 a_{m}\right)^{1 / 2}}\left\|\left(\Omega-\Omega_{0}\right) e_{1}\right\|_{A_{m}} .
\end{aligned}
$$

Proof. It is easy to show that

and so

$$
\Omega_{0}-B \Omega_{0} B=\left(\text { Trace } \Omega_{0}\right) E_{2}=\left(\lambda_{1}+\lambda_{2}\right) E_{2}
$$

$$
\grave{\Omega}_{0}=\frac{1}{2}\left(\Omega_{0}+B \Omega_{0} B\right)=\Omega_{0}-\frac{1}{2}\left(\lambda_{1}+\lambda_{2}\right) E_{2} .
$$

Hence the eigenvalues of the $B$-selfadjoint matrix $\tilde{\Omega}_{0}$ are $\pm \tilde{\mu}_{1}$ where $\tilde{\mu}_{1}=\frac{1}{2}\left(\lambda_{2}-\lambda_{1}\right)$. The eigenvector $\tilde{e}_{1}$ of $\tilde{\Omega}_{0}$ corresponding to $\tilde{\mu}_{1}$ in $e_{2}$ and $B \tilde{e}_{1}=e_{1}$. 
Clearly, $\left(\Lambda, \Lambda^{\prime}\right)$ is a gap in $\sigma_{E}$ if and only if $\left(\Lambda-\frac{1}{2}\left(\lambda_{1}+\lambda_{2}\right)\right.$, $\left.\Lambda^{\prime}-\frac{1}{2}\left(\lambda_{1}+\lambda_{2}\right)\right)$ is a gap in the essential spectrum of the operator $T-$ $\frac{1}{2}\left(\lambda_{1}+\lambda_{2}\right) E_{2}$. We now apply Theorem 1 , with $n=1, j=1$ and $\Omega_{0}, \Omega$ replaced by $\tilde{\Omega}_{0}, \Omega-\frac{1}{2}\left(\lambda_{1}+\lambda_{2}\right) E_{2}$ respectively, to $T-\frac{1}{2}\left(\lambda_{1}+\lambda_{2}\right) E_{2}$. Theorem 2 follows on replacing $\mu_{j}, e_{j}, \Lambda, \Lambda^{\prime}$ in Theorem 1 by $\tilde{\mu}_{1}$, $\tilde{e}_{1}, \Lambda-\frac{1}{2}\left(\lambda_{1}+\lambda_{2}\right)$ and $\Lambda^{\prime}-\frac{1}{2}\left(\lambda_{1}+\lambda_{2}\right)$ respectively and using (2.14).

In Theorem 6.10 of [4] it is shown that if $n=1$ and $\tau$ is assumed to be regular at $a$, then $\sigma_{E} \supset \mathrm{C}\left(\lambda_{1}, \lambda_{2}\right)$ if

$$
\lim _{x \rightarrow \infty} \frac{1}{x} \int_{a}^{x}\left|\Omega(t)-\Omega_{0}\right| d t=0
$$

If in Theorem 2 we restrict our attention to matrices $\Omega$ which are such that $|\Omega(t)|$ is bounded in the intervals $A_{m}$ then our condition (2.11) is clearly stronger than Weidmann's criterion. However without such a restriction, the two criteria are not compatible.

Theorem 2 is evidently a special case of Theorem 1 when $\Omega_{0}$ is $B$-selfadjoint. It is natural to ask whether the $B$-selfadjointness of $\Omega_{0}$ can be omitted in Theorem 1 to obtain an extension of Theorem 2 as it stands. However the method of proof does not allow for a general symmetric matrix $\Omega_{0}$, for, by analogy with the proof of Theorem 2 , this is seen to require an explicit and simple relationship between the eigenvalues and eigenvectors of $\widetilde{\Omega}_{0}$ and $\Omega_{0}$, and an almost random example will serve to eliminate this possibility for $n>1$. If however $\Omega_{0}$ is assumed to be diagonal the method can be applied to obtain an extension of Theorem 2 for $n>1$. We have

TheOREM 3. Let $A_{m}$ be as in Theorem 1. Let $\Omega_{0}$ be a constant real diagonal matrix $d g\left(\lambda_{1}, \cdots, \lambda_{2 n}\right)$ where the eigenvalues $\lambda_{j}$ are not necessarily in increasing order.

If $\lambda_{j} \geqq \lambda_{2 n+1-j}$ and $\frac{1}{2}\left(\Lambda^{\prime}+\Lambda\right) \in \mathrm{C}\left(\lambda_{2 n+1-j}, \lambda_{j}\right)$ then

$$
\Lambda^{\prime}-\Lambda
$$

$$
\leqq 2 \liminf _{m \rightarrow \infty} \frac{1}{\left(2 a_{m}\right)^{1 / 2}}\left\|\left\{\left|\left(\Omega-\Omega_{0}\right) \xi_{j}\right|+\left|\left(\Omega-\Omega_{0}\right) \xi_{2 n+1-j}\right|\right\}\right\|_{A_{m}}
$$

so that

$$
\sigma_{E} \supset \mathrm{C} \bigcap_{j=1}^{2 n}\left(\lambda_{i_{2+1-j}+j}, \hat{\lambda}_{j}\right)
$$

if

$$
\liminf _{m \rightarrow \infty} \frac{1}{\left(2 a_{m}\right)^{12}}\left\|\left|\Omega-\Omega_{0}\right|\right\|_{A_{m}}=0
$$


If $\frac{1}{2}\left(\Lambda^{\prime}+\Lambda\right) \in\left(\lambda_{2 n+1-j}, \lambda_{j}\right)$ then

$$
\Lambda^{\prime} \leqq \lambda_{j}+\liminf _{m \rightarrow \infty} \frac{1}{\left(2 a_{m}\right)^{1 / 2}}\left\|\left(\Omega-\Omega_{0}\right) \xi_{j}\right\|_{A_{m}}
$$

$$
\Lambda \geqq \hat{\lambda}_{2 n+1-j}-\liminf _{m \rightarrow \infty} \frac{1}{\left(2 a_{m}\right)^{1 / 2}}\left\|\left(\Omega-\Omega_{0}\right) \xi_{2 n+1-j}\right\|_{A_{m}} .
$$

Proof. It is readily shown that

and

$$
\Omega_{0}-B \Omega_{0} B=d g_{1 \leqq j \leqq 2 n}\left(\lambda_{j}+\lambda_{2 n+1-j}\right)
$$

$$
\tilde{\Omega}_{0}=\frac{1}{2}\left(\Omega_{0}+B \Omega_{0} B\right)=\frac{1}{2} d g_{1 \leqq j \leqq 2 n}\left(\lambda_{j}-\lambda_{2 n+1-j}\right) .
$$

Hence the eigenvalues of the $B$-selfadjoint matrix $\tilde{\Omega}_{0}$ are $\pm \tilde{\mu}_{j}, j=1,2, \cdots$, $n$, where $\tilde{\mu}_{j}=\frac{1}{2}\left|\lambda_{j}-\lambda_{2 n+1-j}\right|$. The $\tilde{\mu}_{j}$ are in decreasing order if the $\lambda_{j}$ are in increasing order but may not be in any special order otherwise. If $\lambda_{j} \geqq$ $\lambda_{2 n+1-j}$, the eigenvectors $\tilde{e}_{j}, B \tilde{e}_{j}$ of $\tilde{\Omega}_{0}$ corresponding to $\tilde{\mu}_{j},-\tilde{\mu}_{j}$ are $\xi_{j}$ and $-\xi_{2 n+1-j}$. We therefore have

$$
\left\{\tilde{\Omega}_{0}+\frac{1}{2}\left(\lambda_{j}+\lambda_{2 n+1-j}\right)\right\} v=\Omega_{0} v
$$

when $v=\tilde{e}_{j}$ and $B \tilde{e}_{j}$.

From these observations the theorem follows by applying Theorem 1 to $T-\frac{1}{2}\left(\lambda_{j}+\lambda_{2 n+1-j}\right) E_{2 n}$ as in Theorem 2, replacing $\mu_{j}, e_{j}, \Lambda, \Lambda^{\prime}$ in Theorem 1 by $\tilde{\mu}_{j}, \tilde{e}_{j}, \Lambda-\frac{1}{2}\left(\lambda_{j}+\lambda_{2 n+1-j}\right)$ and $\Lambda^{\prime}-\frac{1}{2}\left(\lambda_{j}+\lambda_{2 n+1-j}\right)$ respectively.

Note that if, in Theorem $3, \Omega_{0}$ is a $B$-selfadjoint diagonal matrix with eigenvalues $\pm \mu_{j}, \mu_{1} \leqq \mu_{2} \leqq \cdots \leqq \mu_{n}$ then, in view of (1.1),

$$
\bigcap_{j=1}^{2 n}\left(\lambda_{2 n+1-j}, \hat{\lambda}_{j}\right)=\bigcap_{j=1}^{n}\left(-\mu_{j}, \mu_{j}\right)=\left(-\mu_{1}, \mu_{1}\right) .
$$

It is interesting to note that (2.16) implies that $\sigma_{E}$ contains the whole real line if $\bigcap_{j=1}^{2 n}\left(\lambda_{2 n+1-j}, \lambda_{j}\right)=\varnothing$, a condition which can be fulfilled when $n=1$ only if $\lambda_{1}=\lambda_{2}$ and when $\Omega_{0}$ is $B$-selfadjoint only if $\mu_{1}=0$. This suggests that the dependence of Theorem 3 on the ordering of the eigenvalues of $\Omega_{0}$ along the diagonal, a feature which one is tempted to view with suspicion at first, is indeed of importance. For otherwise the same result would hold with the eigenvalues $\lambda_{j}$ in increasing order and in this case (2.16) only guarantees that $\sigma_{E}$ contains $\mathrm{C}\left(\lambda_{n}, \lambda_{n+1}\right)$ which is not the whole of the real line if $\lambda_{n} \neq \lambda_{n+1}$. Of course, our results do not give any information about the nature of the spectrum inside $\left(\lambda_{n}, \lambda_{n+1}\right)$.

In the final theorem below we assume that some of the eigenvalues of $\Omega$ are large in a certain sense at infinity. 
TheOREM 4. Let $A_{m}$ be as in Theorem 1 . In $A_{m}$ suppose there is defined a real diagonal matrix-valued function $\Omega_{m}=d g\left(\lambda_{1}^{(m)}, \cdots, \lambda_{2 n}^{(m)}\right)$ which satisfies the following conditions:

(i) For some $s, 1 \leqq s \leqq n, \lambda_{s}^{(m)}, \lambda_{2 n+1-s}^{(m)}$ are continuously differentiable functions in $A_{m}$;

(ii) Either

$$
\min _{t \in A_{m}}\left\{\lambda_{s}^{(m)}(t), \lambda_{2 n+1-s}^{(m)}(t)\right\} \rightarrow+\infty
$$

or

$$
\max _{t \in A_{m}}\left\{\lambda_{s}^{(m)}(t), \lambda_{2 n+1-s}^{(m)}(t)\right\} \rightarrow-\infty \text { as } m \rightarrow \infty
$$

(iii) $\left|\lambda_{s}^{(m)}\right| \lambda_{2 n+1-s}^{(m)} \mid$ is bounded as $m \rightarrow \infty$;

(iv) $\left\|\left(\lambda_{i}^{(m)}\right)^{\prime} \mid \lambda_{i}^{(m)}\right\|_{A_{m}}=o\left(a_{m}^{1 / 2}\right)$ as $m \rightarrow \infty$ for $i=s, 2 n+1-s$.

Then

$$
\Lambda^{\prime}-\Lambda
$$

$$
\leqq 2 \liminf _{m \rightarrow \infty} \frac{1}{\left(2 a_{m}\right)^{1 / 2}}\left\|\left\{\left|\left(\Omega-\Omega_{m}\right) \xi_{s}\right|+\left|\left(\Omega 2-\Omega_{m}\right) \xi_{2 n+1-s}\right|\right\}\right\|_{A_{m}} .
$$

Note that in $\Omega_{m}=d g\left(\lambda_{1}^{(m)}, \cdots, \lambda_{2 n}^{(m)}\right)$ the $\lambda_{i}^{(m)}$ are not assumed to be in increasing order.

Proof. In $\varphi^{(m)}(\cdot, \lambda)$ we again take $\lambda=\frac{1}{2}\left(\Lambda^{\prime}+\Lambda\right)$ but this time we choose $f_{m}$ and $v$ (now a $C^{2 n}$-valued function $v^{(m)}$ ) to satisfy

From

$$
\left(i f_{m}^{\prime} B+\Omega_{m}-\lambda\right) v^{(m)}=0 .
$$

$$
\operatorname{det}\left(i f_{m}^{\prime} B+\Omega_{m}-\lambda\right)=\prod_{i=1}^{n}\left\{\left(\lambda_{i}^{(m)}-\lambda\right)\left(\lambda_{2 n+1}^{(m)},-\hat{\lambda}\right)-\left(f_{m}^{\prime}\right)^{2}\right\}
$$

it follows that we may put

$$
f_{m}(t, \lambda)=\int_{c_{m-a_{m}}}^{t}\left\{\left(\lambda_{s}^{(m)}(x)-\lambda\right)\left(\lambda_{2 n+1-s}^{(m)}(x)-\lambda\right)\right\}^{1 \cdot 2} d x, \quad\left|t-c_{m}\right| \leqq a_{m}
$$

and zero otherwise. From (ii), $f_{m}$ is real for any fixed $\lambda$ if $m$ is sufficiently large. As for $v^{(m)}=\left\{v_{i}^{(m)}\right\}$, we put $v_{i}^{(m)}=0$ for $i \neq s, 2 n+1-s$ and

$$
\begin{aligned}
v_{s}^{(m)} & =\frac{\left(\lambda_{2 n+1-s}^{(m)}-\hat{\lambda}\right)^{12}}{\left|\lambda_{s}^{(m)}+\lambda_{2 n+1-s}^{(m)}-2 \lambda\right|^{1 !}}, \\
v_{2 n+1-s}^{(m)} & =\frac{\left(\lambda-\lambda_{s}^{(m)}\right)^{1 / 2}}{\left|\hat{\lambda}_{s}^{(m)}+\hat{\lambda}_{2 n+1 ; s}^{(m)}-2 \lambda\right|^{1,}}
\end{aligned}
$$

As $m \rightarrow x$ we get, from (ii) and (iii),

$$
\left|v^{(m)}\right| \sim 1, \quad\left(v^{(m)}\right)^{\prime}=O\left(\frac{\left(\hat{\lambda}_{s}^{(m)}\right)^{\prime}}{\lambda_{s}^{(m)}}\right)+O\left(\frac{\left(\lambda_{m, n+1}^{(m)}\right)^{\prime}}{\lambda_{i ! n}^{(m)}}\right) .
$$


Hence, since

$$
\begin{aligned}
(\tau-\lambda) \varphi^{(m)}= & \left(i f_{m}^{\prime} B+\Omega_{m}-\lambda\right) \varphi^{(m)} \\
& +k_{m} \exp \left(i f_{m}\right) B\left(u_{m}\left(\cdot-c_{m}\right) v^{(m)}\right)^{\prime}+\left(\Omega-\Omega_{m}\right) \varphi^{(m)}
\end{aligned}
$$

it follows from (iv) and (2.7) that, as $m \rightarrow \infty$,

$$
\begin{aligned}
\left\|(\tau-\lambda) \varphi^{(m)}\right\| \leqq & k_{m}\left\|u_{m}^{\prime}\left(\cdot-c_{m}\right)\right\|_{A_{m}}+k_{m}\left\|\left(v^{(m)}\right)^{\prime}\right\|_{A_{m}} \\
& +k_{m}\left\|\left\{\left|\left(\Omega-\Omega_{m}\right) \xi_{s}\right|+\left|\left(\Omega-\Omega_{m}\right) \xi_{2 n+1-s}\right|\right\}\right\|_{A_{m}} \\
= & o(1)+k_{m}\left\|\left\{\left|\left(\Omega-\Omega_{m}\right) \xi_{s}\right|+\left|\left(\Omega-\Omega_{m}\right) \xi_{2 n+1-s}\right|\right\}\right\|_{A_{m}} .
\end{aligned}
$$

The result now follows as in the proof of Theorem 1 .

\section{REFERENCES}

1. M. S. P. Eastham, On the limit points of the spectrum, J. London Math. Soc. 43 (1968), 253-260. MR 37 \#624.

2. - Gaps in the essential spectrum associated with singular differential operators, Quart. J. Math. Oxford Ser. (2) 18 (1967), 155-168. MR 36 \#451.

3. M. G. Gasymov, The inverse scattering problem for a system of Dirac equations of order 2n, Trudy Moskov. Mat. Obšč. 19 (1968), 41-112=Trans. Moscow Math. Soc. 1968, 41-119. MR 39 \#2418.

4. J. Weidmann, Oszillationsmethoden für Systeme gewöhnlicher Differentialgleichungen, Math. Z. 119 (1971), 349-373.

Department of Purt Mathematics, University College, Cardiff, Wales, UNITED KINGDOM

Department of Mathematics, University of Toronto, Toronto, Ontario, CANADA 